\title{
A Method for Ocean Turbulence Effectiveness Detection and Intelligent Processing Based on Pseudo Dissipation Rate
}

\author{
Dalei Song ${ }^{1}$, Qizhi Yan ${ }^{1}$, Shuxin Wang ${ }^{2}$, Hua Yang ${ }^{2}$, Yuchao Zhu ${ }^{3}$ \\ ${ }^{1}$ College of Engineering, Ocean University of China, Qingdao, 266100, China \\ ${ }^{2}$ College of Information Science and Engineering, Ocean University of China, Qingdao, 266100, China \\ ${ }^{3}$ Physical Oceanography Laboratory, Ocean University of China, Qingdao, 266100, China
}

\begin{abstract}
The exploration and research of ocean turbulence is very important to understand the operational mechanism of ocean circulation, to improve simulation and prediction ability of the global climate changes and circulation. At present, the turbulence instrument includes two types: horizontal turbulent measurement platform and vertical turbulent profiler. In the two types of instrument, the main noise sources are vibrations of platform. And the vibration signal directly affects the quality of turbulence detection. According to the basic principle of turbulence detection, a turbulent data processing method based on pseudo dissipation rate is proposed. This method can improve the intelligent degree of turbulence detection. Compared with turbulent kinetic energy dissipation rate in orders of magnitude, this method can help to find effective turbulence signal. By using the actual observation data, we have verified that method is effective, which verified.
\end{abstract}

Keywords- vibration; turbulence; pseudo dissipation rate.

\section{INTRODUCTION}

Ocean internal turbulence mixing is a very important field for studying. It has become the key factor that restricts the improvement and development of many research fields [1]. The urgent demand for turbulence observation has led to the rapid development of ocean turbulence observation technology. Since 1950s, the ocean observation platform developed from horizontal turbulent measurement platform [2] to vertical turbulent profiler [3], from short-term observation to long-term fixed-point observation. Osborn developed the first vertical profiler that carried shear probe in 1972[4]. In 1997, Lueck developed Tethered Autonomous Microstructure Instrument (TAMI) [5] which had the ability to automatically measured deep ocean turbulence data for a long time.

The vibration of platform will affect the accuracy of turbulence data in the process of turbulence observation, whether horizontal observation platform or vertical observation platform [6]. At present, the domestic and foreign scientists have studied a lot on data noise cancellation method, such as high-pass filter method. In addition, optimizing the design of the platform also can reduce the noise pollution. However, no matter what kind of noise cancellation method is unable to judge the validity of original turbulence data. Therefore, to carry out the turbulence observation instrument intelligent detection mechanism and the study of turbulence observation method has important application value. The strengthening of turbulence intelligent observation can offer more abundant, effective and long-time series observation data for ocean science research. It also can provide strong technical support for ocean turbulence complex features analysis. This paper introduces the turbulence measuring principle, which is based on turbulence observation instrument that developed by Ocean University of China (OUC) [7]. Based on the kiaochow Bay turbulence observation experiment, using attitude sensor (MTI) data, this paper puts forward a method which can calculate the acceleration power spectrum and pseudo dissipation rate. According to the magnitude of pseudo dissipation rate, this method can help to determine the effectiveness of turbulence dissipation rate.

\section{THE BASIS OF OUR METHOD}

\section{A. The principle of turbulence detection}

The principle of turbulence detection is mainly about the shear probe. Shear probe was first put forward by Ribner and Siddon. Osborn improved it and used it for ocean turbulence observation research for the first time. It mainly measures the pulsating quantity of sea water speed. Its main idea is to use the characteristics of piezoelectric ceramic which can convert force signals into electric charge [8]. Piezoelectric ceramics have the characteristics of fast response, high energy density. The rubber head of shear probe feels force. Then force is passed to the piezoelectric ceramic through the cantilever beam. Piezoelectric ceramic generates electrical charge which transmits through wire.

Electrical signals from the shear probe can be represented as:

$$
Q_{p}=\sqrt{2} S U^{2} \sin (2 a)=2 \sqrt{2} S U \cos (a) U \sin (a)=2 \sqrt{2} S W u
$$

$\mathbf{U}$ is the velocity. $\mathbf{W}$ is the velocity along probe axis. $\mathbf{u}$ is the flow rate, which is perpendicular to the probe axis. $\mathbf{S}$ is the sensitivity of the probe. The typical value of $\mathbf{S}$ is

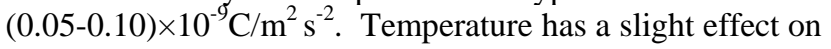
S.

Through the voltage differentiator circuit:

$$
E_{s}=\frac{\partial E_{p}}{\partial t}=2 \sqrt{2} S U \frac{\partial w}{\partial t} \quad E_{p}=Q_{p}
$$

According to Taylor freeze theorem: 


$$
\frac{\partial}{\partial t}=U \frac{\partial}{\partial x}
$$

It can convert the signals from the voltage differentiator into turbulence pulsation shearing signal

$$
\frac{\partial w}{\partial x}=\frac{E_{s}}{2 \sqrt{2} S U^{2}}
$$

Calculation of turbulent kinetic energy dissipation rate

$$
\varepsilon=7.5 v \overline{\left(\frac{\partial u}{\partial z}\right)^{2}}=7.5 v \int_{k_{1}}^{k_{2}} \psi(k) d k
$$

\section{B. The submersible buoy with turbulence instrument}

The experiment was conducted in China Qingdao kiaochow Bay $\left(120^{\circ} 06^{\prime} \sim 120^{\circ} 21^{\prime} \mathrm{E}, 36^{\circ} 00^{\prime} \sim 36^{\circ} 12^{\prime} \mathrm{N}\right)$. Experimental platform is shown in Figure 1.

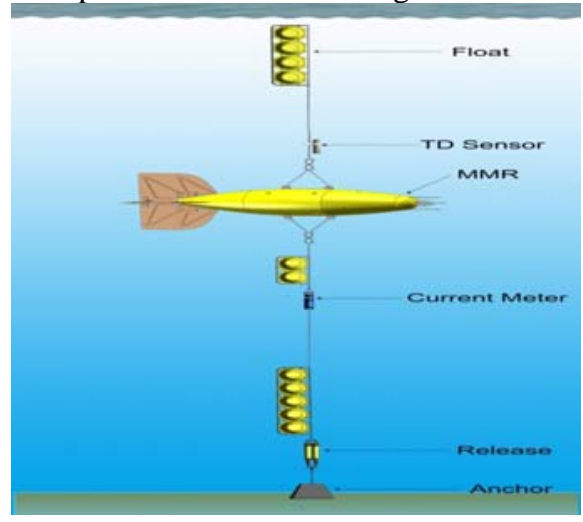

Figure 1. Turbulence observation submersible buoy

The depth of Turbulence observation instrument (MMR): $60-80 \mathrm{~m}$

The velocity of the water: $0.30 \mathrm{~m} / \mathrm{s}$ Temperature: $2.7^{\circ} \mathrm{C}$

\section{PSEUdo Dissipation RATE DATA PROCESSING METHOD}

\section{A. The concept of pseudo dissipation rate}

Shear probe is vibration sensor, which is designed to measure the vibration of the water. When the vibration of the instrument platform is strong enough, the shear probe will perceive it. But normally, the vibration of the instrument platform is very weak. At this time, we use MTI acceleration sensor to measure vibration signal. Then we use the data from MTI to calculate the turbulent kinetic energy dissipation rate. And at this point, the turbulent kinetic energy dissipation rate is the pseudo dissipation rate.

The essence of the pseudo dissipation rate calculation is to replace shear vibration signal with acceleration vibration signals. This paper uses the MTI acceleration sensor. MTI attitude sensor is MEMS structure. Range is $50 \mathrm{~m} / \mathrm{sec}^{2}$.Precision is $0.03 \% \mathrm{FS}$.

\section{B. The calculation of pseudo dissipation rate}

In this paper, we use the MTI attitude Angle and the acceleration signals. Pseudo dissipation rate is calculated:

Step1: Isolate vibration signals which are dynamic acceleration signals through the attitude angle and static acceleration.

$$
\begin{aligned}
& \text { accx }=\text { Acc }+\mathrm{g} * \sin (\text { Pitch } * \pi / 180) ; \\
& \text { accy }=\text { Accy }+\mathrm{g} * \sin (\operatorname{Roll} * \pi / 180) ; \\
& \text { accz }=\text { Accz }+\mathrm{g} * \cos (\operatorname{Roll} * \pi / 180) * \cos (\text { Pitch } * \pi / 180)
\end{aligned}
$$

Step2: The preprocessing of vibration signal. Vibration signals minus its mean value, which eliminates the zero offset of signals.

Step3: Extend the frequency of vibration signals. Sampling frequency of MTI is $120 \mathrm{~Hz}$. We need to expand the frequency of the vibration signals to $1024 \mathrm{~Hz}$.

Step4: The simulation of the vibration signal. Signals divide by the velocity, which can simulate velocity shear signal. When the velocity is $0.5 \mathrm{~m} / \mathrm{s}^{2}$, turbulence signals are the best.

Step5: Calculate the value of a false dissipation rate every four seconds according to the formula (5).

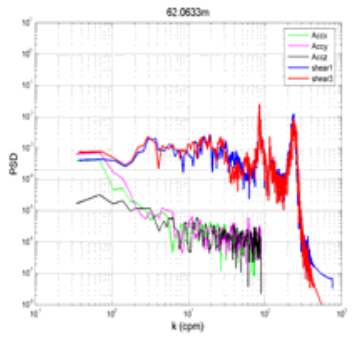

(a)

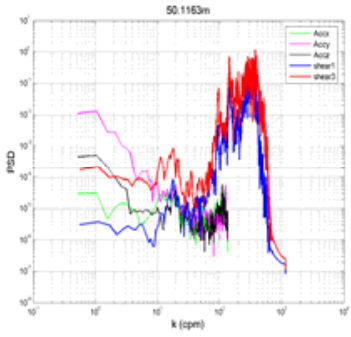

(b)
Figure 2. Shear power spectrum and acceleration power spectrum

\section{THE PROCESSING AND ANALYSIS OF EXPERIMENTAL DATA}

\section{A. The comparison between turbulent shear signal and acceleration signal}

In Figure 2, we compare shear power spectrum and acceleration power spectrum in the wave number space (1$60 \mathrm{cpm}$. As shown in this figure, $\mathrm{X}$ axis is the wave number. $\mathrm{Y}$ axis is the power density. Red and blue curve are shear power spectrum. Black, green and pink curve is acceleration power spectrum. In Figure2 (b), the shear power spectrum and acceleration spectrum overlap, which shows that the vibration of the instrument itself (acceleration signal) seriously affects the shear signal. Obviously, shear signal is not accurate. In Figure2 (a), shear power spectrum and acceleration power spectrum do not overlap. And the difference between shear power spectrum and acceleration power spectrum is $1-2$ orders of magnitude. 


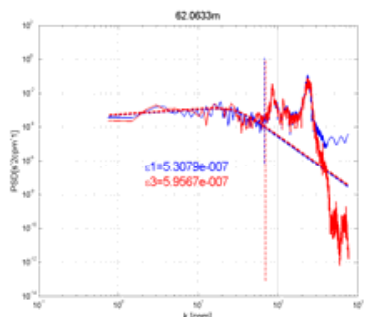

(a)

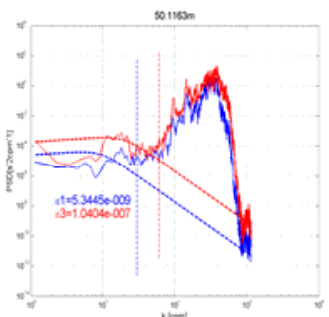

(b)
Figure 3. Shear power spectrum and Nasmyth spectrum

In Figure 3, we compare shear power spectrum and Nasmyth spectrum. As shown in this figure, $X$ axis is the wave number, $\mathrm{Y}$ axis is the power density. Solid lines represent the shear power spectrum. The dotted line was Nasmyth spectrum. Vertical dashed line is the cut-off wave number.

In Figure3 (a), the alignment of shear power spectrum and Nasmyth spectrum is good. Data is the same with Figure 2(a). And in Figure2 (a), shear power spectrum and acceleration power spectrum do not overlap, which shows that the data is good.

In Figure3 (b), alignment is very poor. Data is the same with Figure 2(b). And in Figure2 (b), shear power spectrum and acceleration power spectrum do overlap, which shows that the data is not good. Comparing the two figures, shear signal is more accurate in figure (a).

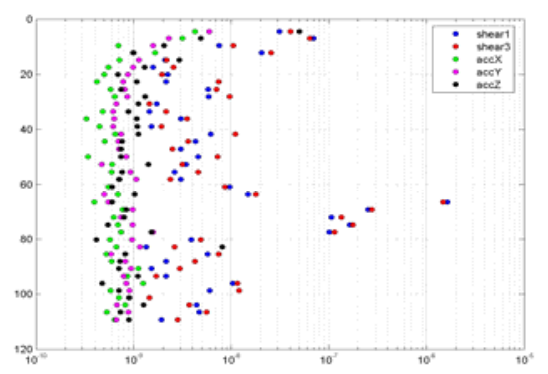

Figure 4. Shear dissipation rate and the acceleration Pseudo dissipation rate

\section{B. The calculation of turbulent shear dissipation rate and acceleration pseudo dissipation rate}

We calculate shear dissipation rate and acceleration pseudo dissipation rate according to the formula (5). Data is collected from the first sea experiment. $\mathrm{X}$ axis is the order of magnitude of dissipation rate. Y axis is depth. Red and blue is shear signal dissipation rate. Black, green and pink is acceleration pseudo dissipation rate.

There are 37 sets of data in this experiment. Using the method of 4.1, we Separate the good and bad data. And good dissipation rate spectrum has 25 according to the formula (5), accounting for the proportion of $65.6 \%$. And the dissipation rate distributes between $10^{-6}$ and $10^{-8}$, scattering. Pseudo dissipation rate is concentrated around $10^{-9}$.
TABLE I. STATISTICS OF DISSIPATION RATE SPECTRUM, THE DISSIPATION RATE AND PSEUDO DISSIPATION RATE

\begin{tabular}{|c|c|c|c|c|c|}
\hline Date & $\begin{array}{c}\text { sea } \\
\text { state }\end{array}$ & $\begin{array}{c}\text { Number } \\
\text { of test }\end{array}$ & $\begin{array}{c}\text { Good } \\
\text { pectrum } \\
\text { percentage }\end{array}$ & $\begin{array}{c}\text { order of } \\
\text { magnitude of } \\
\text { Dissipation } \\
\text { rate }\end{array}$ & $\begin{array}{c}\text { order of } \\
\text { magnitude } \\
\text { of Pseudo } \\
\text { dissipation } \\
\text { rate }\end{array}$ \\
\hline 2012.09 .20 & Level 1 & 1 & $60 \%$ & $10^{-8}$ & $10^{-9}$ \\
\hline 2012.10 .02 & Level 3 & 1 & $70 \%$ & $10^{-8} \sim 10^{-6}$ & $10^{-9}$ \\
\hline 2012.10 .02 & Level 3 & 2 & $70 \%$ & $10^{-7} \sim 10^{-6}$ & $10^{-9}$ \\
\hline 2012.10 .03 & Level 4 & 1 & $65 \%$ & $10^{-8} \sim 10^{-7}$ & $10^{-9}$ \\
\hline 2012.10 .03 & Level 4 & 2 & $62 \%$ & $10^{-8}$ & $10^{-9}$ \\
\hline
\end{tabular}

Table I is the statistics of the experimental data. As can be seen from the table, the order of magnitude of pseudo dissipation rate is stable, basically $10^{-9}$. Good spectrum percentage associated with order of magnitude of dissipation rate between $10^{-8}$ and $10^{-7}$ is higher than good spectrum percentage around $10^{-8}$; Good spectrum percentage associated with order of magnitude of dissipation rate between $10^{-7}$ and $10^{-6}$ is higher than good spectrum percentage between $10^{-8}$ and $10^{-7}$. It can be seen that the percentage of good dissipative spectrum is raised with the improvement of dissipation rate level. So it can be seen that the greater difference between the pseudo dissipation rate and Dissipation rate, the better. At the same time, comparing the second and third set of data, we found dissipation rate level improved, but the increase of good spectrum proportion is not obvious.

According to the above data, we can find that vibration signal will reduce interference to shear signal interference when the difference between the pseudo dissipation rate and dissipation rate is two orders of magnitude.

\section{V.CONCLUSIONS}

The turbulence observation environment is unstable. There are many factors which can cause vibration. The vibration signals will affect the quality of shear signal.

The statistical result of experiment shows that vibration signal can be ignored when the difference between the pseudo dissipation rate and dissipation rate is two orders of magnitude. It can be sure that shear signal quality is very good at this time. Hence, we can use pseudo dissipation rate to determine the effectiveness of the turbulence data. And this method can help scientists find effective shear signal. The method for ocean turbulence effectiveness detection and intelligent processing based on pseudo dissipation rate is effective.

\section{ACKNOWLEDGEMENTS}

The research work was supported by National high technology research and development program (863 program) No. 2012AA090901. 


\section{REFERENCES}

[1] Feng Yue-Yong, Kang Jian-Jun. Shear turbulence data processing technology. Ocean Technology,30(4),pp.19-23, December2007.

[2] Grant H L, Stewart R W, Moilliet A. Turbulence spectra from a tidal channel. J. fluid Mech.12(2),pp.241-268, 1962.

[3] Osborn T R. Estimates of the local rate of vertical diffusion from dissipation measurements. Journal of Physical Oceanography. 10(1),pp.83-89,1980.

[4] Osborn T R. Vertical profiling of velocity microstructure. J. Phys. Oceanogr. 4(1),pp. 109-115, 1974.

[5] Lueck R G, Huang D, Newman D, et al. Turbulence measurement with a moored instrument. Journal of Atmospheric and Oceanic Technology. 14(1), pp. 143-161, 1997
[6] Wang Shu-Xin, Luan Xin, Song Da-Lei. Damping effect of water damping material used in turbulence instruments in submersible buoys. JOURNAL OF VIBRATION AND SHOCK. 33(20),pp.9598,2014.

[7] Song Da-Lei, Sun Jing-Jing, Xue Bing, Tian Chuan. The fixed-point Ocean Turbulence Observation on Submerged Buoy. Intelligent Control, Automatic Detection and High-End Equipment (ICADE), pp.72-77,2012.

[8] Kang Jian-Jun, WU Hai-Qiang, Yang Qing-Xuan. Ocean Turbulence Measurement Technology. Ocean Technology. 26(3), pp. 1923.,2007. 